\title{
Formal guidelines including a pill box improved medication adherence for patients with chronic mental illness
}

\author{
Azrin NH, Teichner G. Evaluation of an instructional program for improving medication compliance for chronically mentally ill \\ outpatients. Behav Res Ther 1998 Sep;36:849-61.
}

\begin{abstract}
Question
Can specific, multicomponent guidelines including a compartmentalised pill box given to patients with mental illness, alone or in the presence of a family member, increase the proportion of patients who comply with their medications?
\end{abstract}

\section{Design \\ Randomised controlled trial with 2 months follow up.}

\section{Setting}

An outpatient mental health clinic in Fort Lauderdale, Florida, USA.

\section{Patients}

49 patients were assessed and 39 completed the study (mean age 38.5 y, $59 \%$ women, $64 \%$ white). Inclusion criteria were chronic mental illness; current use of psychotropic oral medications; $\geqslant 18$ years of age; normal intelligence; living with an adult family member; primary diagnosis of schizophrenia, bipolar I disorder, or major depressive disorder; and not currently abusing alcohol or controlled substances or receiving clozapine.

\section{Intervention}

All interventions lasted 1 hour. Patients in the education only group met with a staff member who read a pamphlet aloud and gave it to the patient. The pamphlet contained information on types of medication, and their action, efficacy, potency, and side effects. Patients in the individual guideline group and the family guideline group received the same printed material that was read aloud and a pill box with 28 compartments (4 time periods/d). The guideline included instruction and skills development on the use of the pill box; taking medication at the same time and place each day; getting prescription refills; setting clinic appointments; alcohol use; side effects; and communication with pharmacists and funding agencies. Family support was the only difference between the 2 guideline groups.

\section{Main outcome measures}

Change in patient adherence with medication (proportion of pills taken) using pill counts and self report; self reported psychiatric symptoms (Symptoms Checklist 90-R); and patients' opinions about the information received.

\section{Main results}

Mean adherence rates were unchanged in the education group $(73 \%$ v 74\%). Adherence increased in both guideline groups (from $70 \%$ to $92 \%$ for patient guideline group and from $76 \%$ to $95 \%$ for the family guideline group) ( $\mathrm{p}<0.05$ for both guideline groups $v$ the education group). The groups did not differ for symptoms or opinions about information received. No demographic variables were identified that predicted medication adherence.

\section{Conclusions}

In patients with chronic mental illness, adherence to medication improved among patients given compartmentalised pill boxes and specific multicomponent guidelines. Similar improvements occurred if patients were alone when given the instructions or if the instructions were given in the presence of an involved family member.

Source of funding: no external funding except donated pill contatiners.

For correspondence: Dr N H Azrin, Center for Psychological Studies, Nova Southeastern University, 3301 College Avenue, Fort Lauderdale, FL 33314, USA. Fax +1 9542623848.

\section{Commentary}

Good compliance with drug treatment is an important factor in preventing readmission to hospital and improving health care. ${ }^{1}$ Previous studies have suggested that continuing effort is needed to develop and maintain innovative approaches that will enable patients to be compliant. ${ }^{2}$ Kemp et al showed that an appropriate intervention helped patients with acute mental illness continue to comply with medications after discharge. ${ }^{3}$ The study by Azrin and Teichner replicates these findings in patients with chronic mental illness after discharge using education and compartmentalised pill boxes. Results were similar with or without involvement of the patient's family.

The study provides a foundation for nurses to develop and evaluate instruc- tional programmes for other patient groups. The study validated self reported adherence with medication with actual pill counts. Patients were evaluated after only 2 months; longer term outcome assessment would have enhanced the credibility and generalisability of the study results. In addition, the evaluation of this multicomponent intervention does not allow us to know which parts of the intervention contributed to the increase in compliance. It would be useful to conduct another study testing pill boxes alone compared with pill boxes plus the additional guidelines and information.

Rose places the onus on practitioners for poor compliance among patients because of inadequate communication and treatment planning. ${ }^{1}$ It is essential therefore that practitioners employ effective interventions to improve compliance as suggested by Haynes et al. ${ }^{2}$ Azrin and Teichner have provided us with an example of a instructional programme that did improve medication compliance.

\section{Catherine Maddock, RN, DipNS Sarum Road Hospital} Winchester, UK

1 Rose NB, editor. Essential psychiatry. Second edition. Oxford: Blackwell Science, 1995.

2 Haynes RB, McKibbon KA, Kanani R, et al. Interventions to assist patients to follow prescriptions for medication. (Cochrane Review, latest version 05 May 1997). In: Cochrane Library. Oxford: Update Software.

3 Kemp R, Hayward P, Applewhaite G, et al. Compliance therapy in psychotic patients: randomised controlled trial. BMJ 1996;312: 345-9. 